\title{
A BP neural network model for predicting the production of a cutter suction dredger
}

\author{
Jinbao Yang ${ }^{1, a^{*}}$, Fusheng $\mathrm{Ni}^{1, \mathrm{~b}}$ and Changyun $\mathrm{Wei}^{1, \mathrm{c}}$ \\ ${ }^{1}$ Engineering Research Center of Dredging Technology of Ministry of Education, Faculty of \\ Mechanical and Electrical Engineering, Hohai University, China \\ ajin_baoyang@163.com, bfushengni@163.com, ${ }^{\text {c }}$.wei@hhu.edu.cn
}

Keywords: Cutter Suction Dredger, Back-Propagation, Neural Networks, Prediction, Production Abstract. In dredging engineering, cutter-suction dredgers are the most widely used dredging equipment in the world. Its production directly determines the efficiency of a dredging project. Therefore, predicting the production of a cutter suction dredger is of considerable importance. This paper presents a BP neural network predictor model. We use Bayesian regularization method to analyze the data from a real cutter suction dredger. Three factors (i.e., the swing speed, the velocity of the hydraulic pipeline transporation, and the work-pressure of the cutter) are considedred in the model to predict the production of the dredger. In addition, we evaluate the proposed model by means of the Matlab neural network toobox.

\section{Introduction}

Dredging engineering is a project that digs water sediment, sand, stone of channel or port areas in order to keep the prescribed scope and depth. It is one of the important projects of water conservancy engineering. Dredging projects have two purposes: one is for agricultural reasons; the second is to dig deeper waterways. Thus, in human economic and social development, dredging engineering still keeps an indispensable status ${ }^{[1,2]}$.

Modern dredging engineering relies mainly on the dredgers. As is known to all, during the construction of dredgers, the monthly production to a great extent determines the engineering benefit. To increase the monthly output for engineering can improve business efficiency ${ }^{[3]}$. It is necessary to study the problem. However, there are many factors that can affect the production, including some regular and irregular factors. Thus, this paper tries to predict production changes with the help of the neural networks.

Artificial Neural Network is a kind of the network system that simulates the human brain information processing mechanism on the basis of the research results of the modern neural network biology. It not only has the general calculation ability of processing the data, but also has the thought of dealing with knowledge, and has the ability of learning and memory.

Back-Propagation neural networks that adjust the connection weights by means of the error gradient descent rule is one of the multilayer feed-forward neural networks. The BP neural network is capable of nonlinear mapping, self-organizing, error feedback adjustment, generalization and fault tolerance. It is able to grasp the inherent laws of something's development. It is meaningful to research the factors of the production and establish the appropriate neural network model based on the data from the cutter suction dredger to analysis the trend. 


\section{The Structure and Algorithm of BP Network}

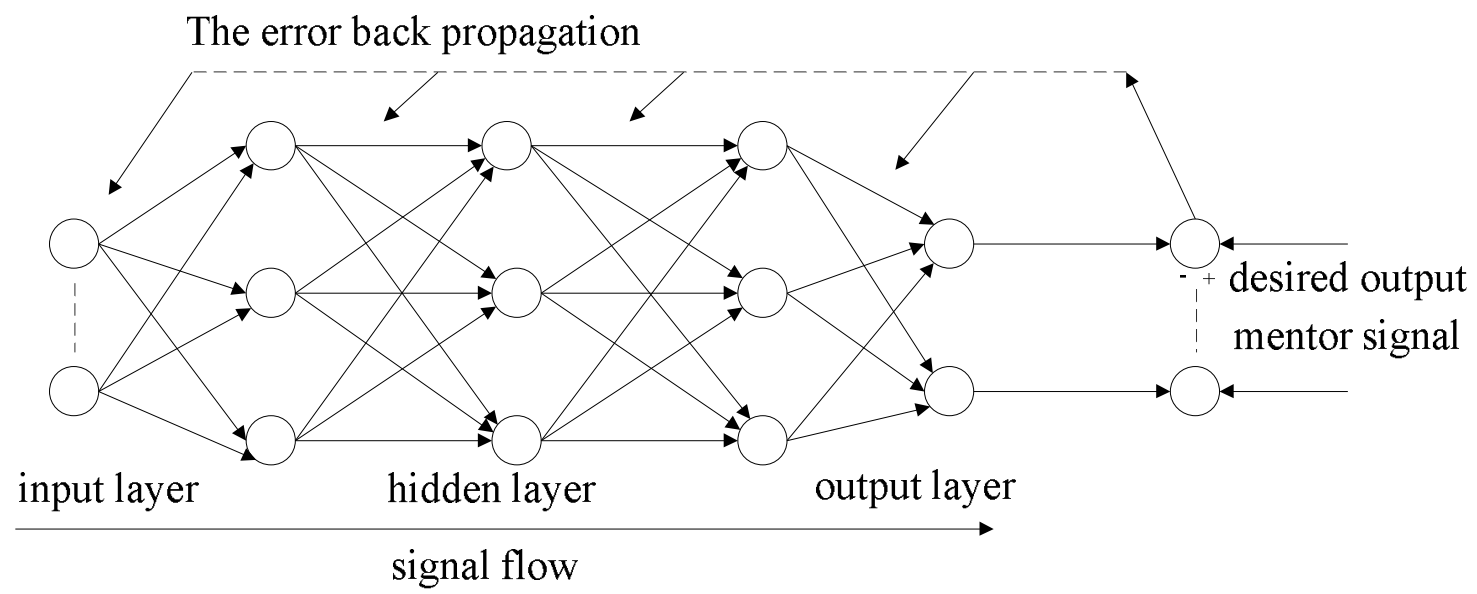

Fig.1. the structure of BP neural network.

Fig. 1 shows that the BP network has the input layer nodes, the output layer nodes, and a layer or multilayer hidden layer nodes. The input layer neurons are responsible for receiving the input information from the outside world and transmitting to the middle layer neurons; the middle layer is the internal information processing layer, and responsible for the information transformation. According to the demand of the ability of information change, the middle layer can be designed for a single hidden layer or multiply hidden layer structure. The last hidden layer transfers information to the output layer. This completes a positive propagation learning process and exports information processing results to the outside after further processing. When the actual output and the desired output is not consistent, the network will enter a stage of the error back propagation. The error through the output layer fixes weights of each layer and back spreads to the hidden layer and the input layer step by step according to error gradient descent methods. The process of the cycle of positive information dissemination and back error propagation is a process that constantly adjusts the values in each layer and trains the neural network. It will continue until the network output error reduces to an acceptable level or reaches a pre-set number of learning.

According to Kosmagoro principle ${ }^{[4]}$ : in the conditions of the reasonable structures and the appropriate weights, a three-layer feed-forward network can approximately close to any continuous function. However, the standard BP algorithm exposes a lot of limitations in the actual application ${ }^{[5]}$. It mainly manifests several aspects in the following: the training process may be caught in a local minimum point; the choice of learning rate lacks effective methods; there aren't effective methods to determine the number of hidden layer neurons, and the function of the network learning and memory is not stable. Due to the defects of the BP neural networks, there are a lot of improved algorithms. The Bayesian regularization algorithm is adopted as follows in this paper.

The error function of the neural network is the mean square error function under normal circumstances. Assume error function $E_{D}$ is:

$$
E_{D}=\sum_{i=1}^{n}\left(t_{i}-a i\right)^{2}
$$

In formula(1), $\mathrm{n}$ is the sample, $t_{i}$ is the desired output, and $a_{i}$ is the network actual output. The meaning of this function is the distance of the expected output and the actual response. Bayesian regularization method adds an $E_{w}$ item which limits approximate function complexity on the basis of formula(1). At this point, the network performance function changes:

$$
\mathrm{F}=\alpha \mathrm{Ew}_{\mathrm{w}}+\beta \mathrm{ED}_{\mathrm{D}}
$$




$$
\mathrm{Ew}_{\mathrm{w}}=\frac{1}{\mathrm{M}} \sum_{\mathrm{i}}^{\mathrm{M}} \omega_{\mathrm{i}}^{2}
$$

In formula(2) and formula(3), $E_{w}$ represents the sum of squares of the network weight, and $\omega_{\mathrm{i}}$ represents the neural network connection weights. $M$ represents the number of the neural network connection power, and $E_{D}$ represents the error function, $\alpha$ and $\beta$ represent the parameters of the objective function. The size of parameter determines the neural network training goals ${ }^{[6]}$.

The Bayesian algorithm automatically normalizes the best error performance adjustment rate, so it has a stronger generalization ability. Even if the network scale increases, it also will not appear the phenomenon of over-adaptation. Thus, we don't need to use the way of trial to determine the best size of the network ${ }^{[7]}$. In the matlab toolbox, the realization of the function of the Bayesian regularization method is trainbr.

\section{The Establishment of the Neural Network Model}

The neural network needs the input layer, the hidden layer and the output layer. We will be starting from the three parts to establish a cutter-suction dredger production model.

\section{Input layer factors}

The swing speed. A transverse device is used to make a cutter-suction dredger dig more widely by swinging in the construction. It can be divided into the left and right lateral movement. The lateral movement of a cutter-suction dredger is done through the transverse winch that is located in the front. The length of the transverse is not only limited by the distance between the work pile and the reamer head, but also restricted by the swing licensing perspective. The swing velocity is determined by the characteristics of the transverse winch, and it has a direct impact on productivity. For different soil, the swing speed is also different. From the above introduction, we can know that the swing velocity is one of the important factors of the production.

The velocity of the hydraulic pipeline transporation. The flow velocity in the pipe is calculated according to different delivery argillaceous sand and the size of the particle diameter of it under the premise of the invariable pipe diameter. Thus, the change of the velocity directly affects the transmission of mud and the change of production ${ }^{[8]}$. When the rotating speed of a underwater pump and the hold pump of a cutter suction dredger keeps constant, its suction capacity can be set to a constant value. The velocity of the hydraulic pipeline transporation changes due to the changes of the cutting soil conditions(i.e., the changes of the soil, the different particle size distribution, the difference of particle's velocity distribution and the slip in the pipe). Therefore, the changes of the flow pattern of solid-liquid two phase flow that is inhaled into the pipe transportation are more complex. However, its changes mainly depend on the flow velocity. The concentrative distribution in the pipe and the velocity distribution of slurry is closely related.

The work-pressure of a cutter. In the working process of a dredger, there is a strong interaction between the reamer and its cutting soil. As the dredging process was done underwater, there are a lot of uncertainties, and it is also one of the widely studied topics at present. In the case of the constant reamer size, the reamer working pressure can reflect the dredging soil features. By observing the work-pressure data of the dredger decision system, we can find that the different working voltage of the reamer reflects the size of the reamer work-pressure. Thus, the reamer working pressure has the influence to the dredging production. This paper uses the swing speed, the velocity of the hydraulic pipeline transporation and the work-pressure of a cutter as the input factors to study their influence to a cutter suction dredger.

Hidden layer. A BP neural network has a difficult problem: it is difficult to determine the number of the hidden layer unit. In the design of a neural network, the number of the hidden layer is usually based on experience and the repeated experiment to determine. There is no an ideal approach to express it. However, the number of the hidden layer and the number of the input layer, the output layer have a direct relationship. Thus, the number of the hidden layer cannot be chosed too much or too little. Otherwise the error may be not the best which makes the fault tolerance poor, and brings down the 
generalization ability. It is very important to choose the hidden layer number. The following three formulas can be used to select the hidden layer unit number.

(1) $\mathrm{n}_{1}=\sqrt{\mathrm{n}+\mathrm{m}}+\mathrm{a}$, the $\mathrm{m}$ is for the output neuron number and the $\mathrm{n}$ is for the input neuron number, and $\mathrm{a}$ is constant between 1 to 10 .

(2) $\mathrm{n}_{1}=\log _{2} \mathrm{n}$, the $\mathrm{n}$ is for the input unit number.

(3) the Kolmogorov theory is $n_{1}=2 n+1$, and the $n$ is for the input unit number.

An another way can also be used to determine the number of the hidden units. First make the number of the hidden unit variable, or add enough hidden units. The system gets rid of those units by learning which do not work out, until it doesn't shrink. We can also put the fewer neurons in the beginning. If the system doesn't succeed after learning to the certain times, we could increase the number of the hidden units until it achieves a reasonable value.

In this paper, we use three input factors which are described above. According to the first formula of the hidden layer we can calculate that it is optimal in range of 3 to 12 for the numbers of the hidden layers when the input factor is three.

Output layer factor. The production rate represents mining earthwork quantity per hour and reflects the changes of the production. During the dredging process we pursue high yield, thus the production rate is put as the output factor.

\section{Simulation and Prediction}

According to the BP neural network introduced above, we choose that the training sample is 1 seconds to 1500 seconds and the test data is 1500 seconds to 1700 seconds and 1700 seconds to 1900 seconds. The hided number sets to a layer and the number of nodes is 10 which can be appropriately adjusted according to the error. The transfer function of the hidden layer is logsig function and the output layer is purelin function. The expect error is 0.01 and the learning rate is 0.1 . The training function is the Bayesian regularization method. The number of the training is 10000 . We use Matlab neural network tools to make this model. The result of the prediction is shown in Fig.2:

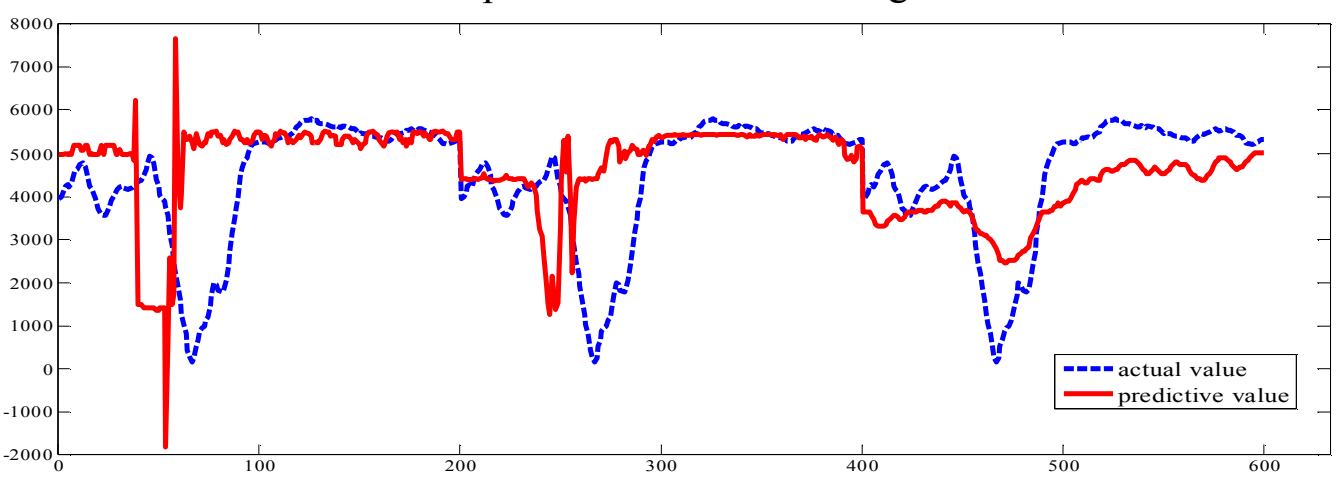

(a)

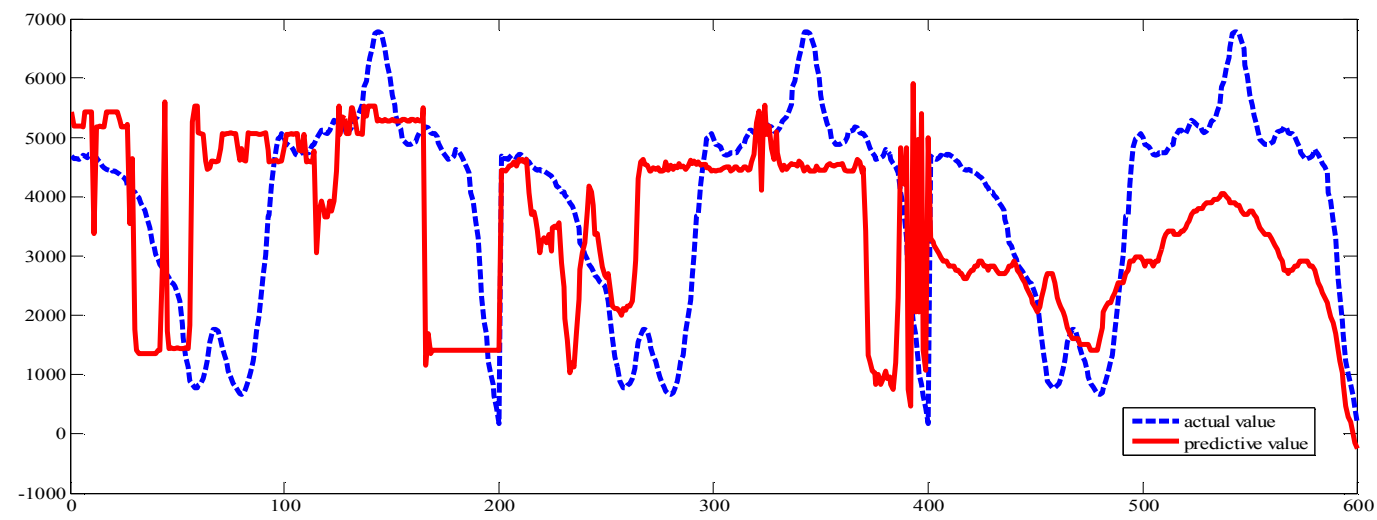

(b)

Fig.2. the yield prediction of the three factors. 
From Fig.2, the trend of the yield prediction of the input factors which is the swing speed, the velocity of the hydraulic pipeline transporation and the work pressure of a cutter is consistent to the actual output. It shows that the BP network model can be used for the research of a cutter suction dredger' production, and has a good prediction effect. There are some time delay and lag in Fig.2. It is mainly because there are certain pipeline lengths between the reamer head and productivity meter. When the reamer begins cutting soil, the slurry in the pipeline has not yet to reach the production installation location. This requires a period of time, which causes time delay phenomenon. When the reamer stops cutting soil, the residual mud in the pipe will still pass through the production installation location. The productivity meter will still calculate the production that causes time lag phenomenon. There are some abnormal points in about 50 seconds in Fig.2a that may be due to the noise existing in the sample data. The data that collected from the construction site often have the abnormal datas, including the data of the reamer stop running and the underwater pump stop running and so on. The error of these data and normal data is usually bigger. The noise data will make effect to a BP neural network prediction. At the same time the multiple factors as input will be more prone to make the error.This shows the influence factors of the parameter prediction remains to be discovered.

\section{Conclusion}

This paper uses neural network's self-learning ability to study the production characteristics of a cutter suction dredger. The main results are listed as follows:

(1) This paper is based on the Bayesian regularization algorithm as the training function of the BP neural network. It has good generalization ability and has certain reference significance to the prediction of the production of a cutter-suction dredger.

(2) The influence of three factors (i.e., the swing speed, the velocity of the hydraulic pipeline transporation and the work-pressure of a cutter) on the production gets well prediction. In addition, the trend of predictive value and actual value keeps consistent. Thus, the proposed BP neural network model can well predict the output of a cutter-suction dredger.

However, the propsoed model remains to be perfect. If we can solve the problem of time delay, the prediction of dredger's output will be more accurate. In addition, the increase of the factors that may influence the production can get better results.

\section{References}

[1] Shoujin Liu, Cutter-suction dredger construction and management, China water conservancy and hydropower press, BeiJing, 2005.

[2] Liusi Yan, River dredging engineering construction technology development at home and abroad, China's water resources and hydropower, 2006.12.

[3] Wangjin Wang, Guangjun Zai, Increase production of cutter-suction dredger, The Yangtze river waterway engineering in wuhan, Wuhan, China, 2007.07.

[4] Ougeng Liu, Multilayer feedforward neural network structure research: Zhuzhou institute of technology, 2003.11.

[5] Liqun Han, The theory, design and application of artificial neural network, Chemical industry press, Beijing, 2007.7, pp.54-55.

[6] Xujun Li, Application of the BP neural network of Bayesian regularization in economic forecasting, Central China normal university, 2009

[7] Kaili Zhou, Yaohong Kang, Neural network model and matlab simulation program design, Tsinghua University press, Beijing, 2005.7, pp.2-4. 
[8] Changlin Sun, Design and calculation of mud pump and mud system, The Yangtze river ship design institute, 2000.11 . 